\title{
Classical relativistic system of $N$ charges. Hamiltonian description, forms of dynamics, and partition function
}

\author{
A.Duviryak, A.Nazarenko, V.Tretyak \\ Institute for Condensed Matter Physics \\ of the National Academy of Sciences of Ukraine, \\ 1 Svientsitskii Str., 79011 Lviv, Ukraine
}

Received October 3, 2000

\begin{abstract}
The procedure of reducing canonical field degrees of freedom for a system of charged particles plus field in the constrained Hamiltonian formalism is elaborated up to the first order in the coupling constant expansion. The canonical realization of the Poincare algebra in the terms of particle variables is found. The relation between covariant and physical particle variables in the Hamiltonian description is written. The system of particles interacting by means of scalar and vector massive fields is also considered. The first order approximation in $c^{-2}$ is examined. An application to calculating the relativistic partition function of an interacting particle system is discussed.
\end{abstract}

Key words: classical relativistic mechanics, forms of relativistic dynamics, relativistic statistical mechanics, charged particles

PACS: 03.30. $+p, 05.20 .-y$

\section{Introduction}

During a long period of time, the development of relativistic statistical mechanics was limited by insufficient understanding of the relativistic classical and quantum description of the systems with finitely many particles. At present, the classical relativistic mechanics of $N$-particle system with the direct interaction is formulated in a consistent way within various formalisms and approaches. These formulations are based on the Poincaré-invariance conditions; the relation of the general expressions for interaction potentials with the concrete field-theoretical model can be performed either by means of several approximation (e.g., Darwin's Lagrangian in electrodynamics), or with the use of the Fokker-type action integrals (see, e.g., [1]).

The different formalisms of the classical relativistic mechanics are more or less 
equivalent, although the Lagrangian formalism has some advantages in its conceptual simplicity and the direct relations with the field-theoretical models [1,2]. But statistical description of the interacting particle system is more transparent in the Hamiltonian formalism. The transition from Lagrangian to Hamiltonian description in the classical relativistic dynamics is not simple or direct and demands the use of various approximations. On the other hand, it is natural to try constructing the Hamiltonian description of an interacting particle system starting from the Hamiltonian formalism for the "particle plus field" system and then eliminating the field degrees of freedom. Such a program was discussed in the illuminating series of papers by Lusanna with coworkers (see [3]).

Here we present a simpler approach $[4,5]$ which uses the geometrical forms of dynamics [2] to impose the gauge fixing conditions concerning the chronometrical invariance of action. In section 2 we consider the constrained Hamiltonian description of charged particles with electromagnetic fields and the canonical transformation which isolate nonphysical (gauge) degrees of freedom of the electromagnetic fields. We also consider the massive scalar and vector interactions and obtain the generators of time evolution and Lorentz transformations on the physical phase space. The elimination of the field degrees of freedom is discussed in section 3 within the linear approximation in the coupling constant. We obtain the canonical generators of the Poincaré group for the interactions considered. We demonstrate that the first order approximation in $c^{-2}$ agrees with the well known results of various approaches. An application to calculating the relativistic partition function of an interacting particle system is discussed in section 4 .

\section{Hamiltonian description of the "field plus particle" system}

At the beginning we briefly outline the main steps of the Hamiltonian description of a system of $N$ point particles with electromagnetic interaction [5]. The particles are described by their world lines in the Minkowski space-time ${ }^{1} \gamma_{a}: \tau \mapsto x_{a}^{\mu}(\tau)$. An interaction between charges is mediated by an electromagnetic field $F_{\mu \nu}(x)=$ $\partial_{\mu} A_{\nu}(x)-\partial_{\nu} A_{\mu}(x)$ with the electromagnetic potential $A_{\mu}(x) ; \partial_{\nu} \equiv \partial / \partial x^{\nu}$. An action for the system is

$$
S=-\sum_{a=1}^{N} \int \mathrm{d} \tau_{a}\left\{m_{a} \sqrt{u_{a}^{2}\left(\tau_{a}\right)}+e_{a} u_{a}^{\nu}\left(\tau_{a}\right) A_{\nu}\left[x_{a}\left(\tau_{a}\right)\right]\right\}-\int \mathrm{d}^{4} x F^{2},
$$

where $m_{a}$ and $e_{a}$ are the mass and the charge of particle $a$, respectively, $F^{2} \equiv$ $(1 / 4) F_{\lambda \sigma}(x) F^{\lambda \sigma}(x)$, and $u_{a}^{\mu}\left(\tau_{a}\right)=\mathrm{d} x_{a}^{\mu}\left(\tau_{a}\right) / \mathrm{d} \tau_{a}$. The action is manifestly invariant under two types of gauge transformations: reparametrization of the particle world

\footnotetext{
${ }^{1}$ The Minkowski space-time is endowed with a metric $\left\|\eta_{\mu \nu}\right\|=\operatorname{diag}(1,-1,-1,-1)$. The Greek indices $\mu, \nu, \ldots$ run from 0 to 3 ; the Roman indices from the middle of alphabet, $i, j, k, \ldots$ run from 1 to 3 and both types of indices are subject of the summation convention. The Roman indices from the beginning of alphabet, $a, b$, label the particles and run from 1 to $N$. The sum over such indices is indicated explicitly.
} 
lines

$$
\tau_{a} \mapsto \phi\left(\tau_{a}\right), \quad \phi^{\prime}>0,
$$

and ordinary gauge transformation of the electromagnetic potential

$$
A_{\mu} \mapsto A_{\mu}+\partial_{\mu} \Lambda
$$

Moreover, action (2.1) is invariant under (global) transformations of the Poincaré group; this invariance results in the conservation of the symmetric energy-momentum tensor $[6]$ :

$$
\begin{gathered}
\theta^{\mu \nu}(x)=\sum_{a=1}^{N} \int m_{a} \frac{u_{a}^{\mu}\left(\tau_{a}\right) u_{a}^{\nu}\left(\tau_{a}\right)}{\sqrt{u_{a}^{2}\left(\tau_{a}\right)}} \delta^{4}\left(x-x_{a}\left(\tau_{a}\right)\right) \mathrm{d} \tau_{a}-F^{\mu \lambda} F_{\lambda}^{\nu}+\eta^{\mu \nu} F^{2}, \\
\theta^{\mu \nu}(x)=\theta^{\nu \mu}(x), \quad \partial_{\nu} \theta^{\mu \nu}(x)=0 .
\end{gathered}
$$

We fix the freedom in the parametrization of particle world lines by choosing the form of relativistic dynamics, which is specified by one-parameter family $\left\{\Sigma_{t} \mid t \in \mathbb{R}\right\}$ of space-like or isotropic hypersurfaces $\Sigma_{t}=\left\{x \in \mathbb{M}_{4} \mid \sigma(x)=t\right\}$ foliating the Minkowski space-time (see [2]). Because the hypersurface equation $\sigma(x)=t$ can be solved with respect to $x^{0}$ in the form:

$$
x^{0}=f(t, \mathbf{x}), \quad \mathbf{x}=\left(x^{1}, x^{2}, x^{3}\right),
$$

the functions $x^{i}=x_{a}^{i}(t), i=1,2,3$, completely determine the parametric equations of the particle world lines in a given form of dynamics:

$$
x^{0}=f\left(t, \mathbf{x}_{a}(t)\right), \quad x^{i}=x_{a}^{i}(t) .
$$

The variable $t$ serves as a common evolution parameter of the system.

Now we can use the definition of the form of dynamics as a gauge fixing condition and put action (2.1) into a single-time form [5]

$$
S=\int \mathrm{d} t L
$$

with Lagrangian $L(t)$ depending on the functions $\mathbf{x}_{a}(t), A^{\mu}(t, \mathbf{x})$ and their first order derivatives with respect to evolution parameter, $\dot{\mathbf{x}}_{a}(t)=\mathrm{d} \mathbf{x}_{a}(t) / \mathrm{d} t$ and $\dot{A}^{\mu}(t, \mathbf{x})$. The conservation of the energy-momentum tensor (2.4) gives us ten conserved quantities in a given form of dynamics:

$$
P^{\mu}=\int_{\Sigma_{t}} \theta^{\mu \nu} d \sigma_{\nu}, \quad M^{\mu \nu}=\int_{\Sigma_{t}}\left(x^{\mu} \theta^{\nu \rho}-x^{\nu} \theta^{\mu \rho}\right) \mathrm{d} \sigma_{\rho} .
$$

The single-time Lagrangian $L$ is invariant under gauge transformation (2.3) and leads to the constrained Hamiltonian description. As it has been discussed in [5], the structure of the corresponding constraints depend on the form of dynamics. In the following we confine ourselves to the most common case of the instant form of 
dynamics $\left(x^{0}=t\right)$. The Lagrangian function in this form of dynamics is represented by:

$$
L=-\sum_{a=1}^{N}\left\{m_{a} \sqrt{1-\dot{\mathbf{x}}_{a}^{2}}+e_{a}\left[A_{0}\left(t, \mathbf{x}_{a}\right)+\dot{x}_{a}^{i} A_{i}\left(t, \mathbf{x}_{a}\right)\right]\right\}-\frac{1}{4} \int\left(2 e_{i} e^{i}+F_{i j} F^{i j}\right) \mathrm{d}^{3} x
$$

where $F_{i j}=A_{j, i}-A_{i, j}$ and $e_{i}=A_{0, i}-\dot{A}_{i}$.

In the Hamiltonian formulation of our system we start with canonical variables $x_{a}^{i}(t), A_{\mu}(t, \mathbf{x})$ and conjugated momenta $p_{a i}(t), E^{\mu}(t, \mathbf{x})$ which are subject of the first class constraints [7]

$$
E^{0} \approx 0, \quad \Gamma \equiv \varrho-\partial_{i} E^{i} \approx 0,
$$

where $\approx$ means "weak equality" in the sense of Dirac and $\varrho(t, \mathbf{x})=\sum_{a=1}^{N} e_{a} \delta^{3}\left(\mathbf{x}-\mathbf{x}_{a}(t)\right)$ is a charge density. Then we perform the canonical transformation that dissect the field phase space into the physical part described by the gauge invariant variables $a_{\alpha}=\left(\delta_{\alpha}^{i}-\delta_{3}^{i} \partial_{\alpha} / \partial_{3}\right) A_{i}, E^{\alpha} ; \alpha=1,2$, and unphysical part parametrized by the canonical pairs $(Q, \Gamma)$ and $\left(A_{0}, E^{0}\right)$.

The time evolution of the physical degrees of freedom is generated by the Hamiltonian

$$
H=\sum_{a=1}^{N} \sqrt{m_{a}^{2}+\left[\mathbf{p}_{a}-e_{a} \mathbf{A}_{\perp}\left(\mathbf{x}_{a}\right)\right]^{2}}-\frac{1}{2} \int\left(A_{i}^{\perp} \Delta A_{i}^{\perp}-E_{\perp}^{i} E_{\perp}^{i}+\varrho \Delta^{-1} \varrho\right) \mathrm{d}^{3} x,
$$

where

$$
E_{\perp}^{i}=\left(\delta_{\alpha}^{i}-\delta_{3}^{i} \partial_{\alpha} / \partial_{3}\right) E^{\alpha}, \quad A_{i}^{\perp}=\left(\delta_{i}^{\alpha}+\partial_{i} \Delta^{-1} \partial^{\alpha}\right) a_{\alpha} .
$$

Inverse differential operators are defined by the relations

$$
1 / \partial_{3} \delta^{3}(\mathbf{x})=(1 / 2) \delta\left(x^{1}\right) \delta\left(x^{2}\right) \operatorname{sgn}\left(x^{3}\right), \quad \Delta^{-1} \delta^{3}(\mathbf{x})=-1 /(4 \pi|\mathbf{x}|) .
$$

Conserved quantities (2.9) being reexpressed in terms of canonical variables determine the canonical realization of the Poincaré group. On the physical subspace the generator $P^{0}$ coincide with the Hamiltonian (2.12), and the generator of the Lorentz transformation is given by

$$
\begin{aligned}
M^{k 0}= & \sum_{a=1}^{N}\left\{x_{a}^{k} \sqrt{m_{a}^{2}+\left[\mathbf{p}_{a}-e_{a} \mathbf{A}_{\perp}\left(\mathbf{x}_{a}\right)\right]^{2}}-t p_{a}^{k}\right\}-\frac{1}{2} \int x^{k} \varrho \Delta^{-1} \varrho \mathrm{d}^{3} x \\
& +\int x^{k}\left(\frac{1}{4} F_{i j}^{\perp} F_{i j}^{\perp}+\frac{1}{2} E_{\perp}^{i} E_{\perp}^{i}+E_{\perp}^{l} \partial_{l} \Delta^{-1} \varrho\right) \mathrm{d}^{3} x-t \int E_{\perp}^{l} \partial^{k} A_{l}^{\perp} \mathrm{d}^{3} x .
\end{aligned}
$$

where $F_{i j}^{\perp}=A_{j, i}^{\perp}-A_{i, j}^{\perp}$.

It is instructive to consider in a similar manner the Hamiltonian description of the system of particles with massive vector and scalar interactions. So, in the first case we have a system described by the action that differs from (2.1) by a massive 
term $\frac{1}{2} \mu^{2} A^{\nu} A_{\nu}$. The instant form of Hamiltonian description of the system is based on the canonical variables $x_{a}^{i}(t), A_{\mu}(t, \mathbf{x})$ and $p_{a i}(t), E^{\mu}(t, \mathbf{x})$ with a pair of the second class constraints

$$
E^{0} \approx 0, \quad \Gamma-\mu^{2} A_{0} \approx 0,
$$

which can be excluded by means of the Dirac bracket. The canonical Hamiltonian is

$$
\begin{aligned}
H= & \sum_{a=1}^{N} \sqrt{m_{a}^{2}+\left[\mathbf{p}_{a}-e_{a} \mathbf{A}\left(t, \mathbf{x}_{a}\right)\right]^{2}} \\
& +\int\left[\frac{1}{4} F_{i j} F_{i j}+\frac{1}{2} E^{i} E^{i}-\frac{1}{2} \mu^{2} A_{i} A^{i}+A_{0}\left(\Gamma-\frac{1}{2} \mu^{2} A_{0}\right)\right] \mathrm{d}^{3} x .
\end{aligned}
$$

After exclusion of the constraints (2.16) one obtains for the boost generator

$$
\begin{aligned}
M^{k 0}= & \sum_{a=1}^{N}\left\{x_{a}^{k} \sqrt{m_{a}^{2}+\left[\mathbf{p}_{a}-e_{a} \mathbf{A}\left(t, \mathbf{x}_{a}\right)\right]^{2}}-t p_{a}^{k}\right\} \\
& +\int x^{k}\left[\frac{1}{4} F_{i j} F_{i j}+\frac{1}{2} E^{i} E^{i}-\frac{1}{2} \mu^{2} A_{i} A^{i}+\frac{1}{2 \mu^{2}} \Gamma^{2}\right] \mathrm{d}^{3} x \\
& -t \int\left[E^{j} \partial^{k} A_{j}-\frac{1}{2} \mu^{2} A^{k} \Gamma\right] \mathrm{d}^{3} x .
\end{aligned}
$$

In the case of a system of particles interacting by means of the scalar field $\varphi(x)$ we find the standard Hamiltonian description without constraints with the Hamiltonian

$$
H=\sum_{a=1}^{N} \sqrt{\mathbf{p}_{a}^{2}+\left[m_{a}-e_{a} \varphi\left(t, \mathbf{x}_{a}\right)\right]^{2}}+\frac{1}{2} \int\left[\pi^{2}+(\boldsymbol{\nabla} \varphi)^{2}+\mu^{2} \varphi^{2}\right] \mathrm{d}^{3} x
$$

and the boost generator

$$
\begin{aligned}
M^{k 0}= & \sum_{a=1}^{N}\left\{x_{a}^{k} \sqrt{\mathbf{p}_{a}^{2}+\left[m_{a}-e_{a} \varphi\left(t, \mathbf{x}_{a}\right)\right]^{2}}-t p_{a}^{k}\right\} \\
& +\frac{1}{2} \int x^{k}\left[\pi^{2}+(\boldsymbol{\nabla} \varphi)^{2}+\mu^{2} \varphi^{2}\right] \mathrm{d}^{3} x-t \int \pi \partial^{k} \varphi \mathrm{d}^{3} x .
\end{aligned}
$$

We will see that after elimination of the field degrees of freedom all the three considered cases give the canonical generators of a similar structure.

\section{Elimination of the field degrees of freedom}

Now we are interested in elimination of the physical field degrees of freedom. As a result, we shall obtain the description of our system in the terms of particle variables only. Such a reformulation is especially effective when the free radiation is not essential. 
Our procedure of the field reduction has got three steps [8]. First, we must find a solution of the field equations of motion. In the Hamiltonian mechanics the field equations are non-linear, so we use an approximation which is based on the coupling constant expansion. In general, the problem of choosing the Green's function arises in this approach. But in the first-order (linear) approximation in the coupling constant the advanced, retarded, or symmetric solutions coincide. We use here the timesymmetric Green's function $G\left(x^{2}\right)=G\left(x_{0}^{2}-\mathbf{x}^{2}\right)$. It is well known [1], that the Green's function determines the nonrelativistic potential $u(r)$ :

$$
u(r)=\int \mathrm{d} \alpha G\left(\alpha^{2}-r^{2}\right)
$$

The general solution of the field equations is a sum of the source free field $A_{s}^{\mathrm{rad}}$ ( $s$ is the number of the physical field components), which satisfies the homogeneous equation, and the solution of the inhomogeneous equation $\mathcal{A}_{s}$ in the terms of canonical particle variables.

Second, we perform a canonical transformation [8]:

$$
\begin{aligned}
& A_{s}=A_{s}^{\mathrm{rad}}+\mathcal{A}_{s}, \quad E^{s}=E_{\mathrm{rad}}^{s}+\mathcal{E}^{s}, \\
& x_{a}^{i}=q_{a}^{i}+\int\left[\left(A_{s}^{\mathrm{rad}}+\frac{1}{2} \mathcal{A}_{s}\right) \frac{\partial \mathcal{E}^{s}}{\partial k_{a i}}-\left(E_{\mathrm{rad}}^{s}+\frac{1}{2} \mathcal{E}^{s}\right) \frac{\partial \mathcal{A}_{s}}{\partial k_{a i}}\right] \mathrm{d}^{3} x, \\
& p_{a i}=k_{a i}-\int\left[\left(A_{s}^{\mathrm{rad}}+\frac{1}{2} \mathcal{A}_{s}\right) \frac{\partial \mathcal{E}^{s}}{\partial q_{a}^{i}}-\left(E_{\mathrm{rad}}^{s}+\frac{1}{2} \mathcal{E}^{s}\right) \frac{\partial \mathcal{A}_{k}^{\perp}}{\partial q_{a}^{i}}\right] \mathrm{d}^{3} x,
\end{aligned}
$$

after that the free field terms $\left(A_{s}^{\mathrm{rad}}, E_{\mathrm{rad}}^{s}\right)$ become the new canonical variables. It is assumed in our problem that the field hasn't got its own degrees of freedom, so third step consists in elimination of the field variables by means of constraints

$$
A_{s}^{\mathrm{rad}} \approx 0, \quad E_{\mathrm{rad}}^{s} \approx 0
$$

We find the Dirac bracket, which coincides with the particle Poisson bracket $\left\{q_{a}^{i}, k_{b j}\right\}=\delta_{a b} \delta_{j}^{i}$. The canonical generators of the Poincaré group for the considered interactions in the linear approximation are

$$
\begin{aligned}
& H=c \sum_{a=1}^{N} k_{a}^{0}+\frac{c}{2} \sum_{a, b=1}^{N} e_{a} e_{b} \frac{f\left(\omega_{a b}\right)}{k_{a}^{0}} u\left(\rho_{a b}\right), \quad k_{a}^{0}=\sqrt{m_{a}^{2} c^{2}+\mathbf{k}_{a}^{2}}, \\
& P^{k}=\sum_{a=1}^{N} k_{a}^{k}, \quad M^{i j}=\sum_{a=1}^{N}\left(q_{a}^{i} k_{a}^{j}-q_{a}^{j} k_{a}^{i}\right), \\
& M^{k 0}=\sum_{a=1}^{N}\left(\frac{q_{a}^{k}}{c} k_{a}^{0}-t k_{a}^{k}\right)+\frac{1}{2 c} \sum_{a, b=1}^{N} e_{a} e_{b} q_{b}^{k} \frac{f\left(\omega_{a b}\right)}{k_{a}^{0}} u\left(\rho_{a b}\right),
\end{aligned}
$$

where the prime over sum denotes that $a \neq b(a=b$ terms is excluded by means of mass renormalization); $\rho_{a b}^{2}=q_{a b}^{2}+\left(\mathbf{k}_{a} \mathbf{q}_{a b} / k_{a}^{0}\right)^{2}, \mathbf{q}_{a b}=\mathbf{q}_{a}-\mathbf{q}_{b}, q_{a b}=\left|\mathbf{q}_{a b}\right|, \omega_{a b}=$ 
$k_{a}^{\mu} k_{b \mu} / m_{a} m_{b} c^{2}$, and $f(\omega)=1$ for the scalar interaction and $f(\omega)=\omega$ for the vector interaction. It can be easily demonstrated, that the expressions (3.6)-(3.8) satisfy the commutation relations of the Poincaré group in a given approximation with arbitrary functions $u(r)$ and $f(\omega)$.

According to (3.3), the covariant particle positions $x_{a}^{i}$ are connected with the canonical variables as

$$
x_{a}^{i}=q_{a}^{i}+\frac{1}{2} \int\left[\mathcal{A}_{s} \frac{\partial \mathcal{E}^{s}}{\partial k_{a i}}-\mathcal{E}^{s} \frac{\partial \mathcal{A}_{s}}{\partial k_{a i}}\right] \mathrm{d}^{3} x .
$$

It can be verified directly that in a given approximation the expression (3.9) satisfies the world line condition

$$
\left\{x_{a}^{i}, M^{k 0}\right\}=x_{a}^{k}\left\{x_{a}^{i}, H\right\}-t \delta^{i k} .
$$

The Poisson brackets between particle positions do not vanish,

$$
\left\{x_{a}^{i}, x_{b}^{j}\right\}=\int\left(\frac{\partial \mathcal{A}_{s}}{\partial k_{b j}} \frac{\partial \mathcal{E}^{s}}{\partial k_{a i}}-\frac{\partial \mathcal{E}^{s}}{\partial k_{b j}} \frac{\partial \mathcal{A}_{s}}{\partial k_{a i}}\right) \mathrm{d}^{3} x,
$$

in a full agreement with the famous no-interaction theorem [9].

Let us examine the generators (3.6), (3.8) up to $c^{-2}$ approximation. Using the first order expansions

$$
u\left(\rho_{a b}\right)=u\left(q_{a b}\right)+\frac{\left(\mathbf{q}_{a b} \mathbf{k}_{a}\right)^{2}}{2 q_{a b} m_{a}^{2} c^{2}} \frac{\mathrm{d} u\left(q_{a b}\right)}{\mathrm{d} q_{a b}}, \quad f\left(\omega_{a b}\right)=1+\frac{f^{\prime}(0)}{2 c^{2}}\left(\frac{\mathbf{k}_{a}}{m_{a}}-\frac{\mathbf{k}_{b}}{m_{b}}\right)^{2}
$$

and performing the canonical transformation generated by the function

$$
\Lambda=\frac{1}{4 c^{2}} \sum_{a<b}^{N} e_{a} e_{b} u\left(q_{a b}\right)\left[\mathbf{q}_{a b}\left(\frac{\mathbf{k}_{a}}{m_{a}}-\frac{\mathbf{k}_{b}}{m_{b}}\right)\right]
$$

we obtain the expressions

$$
\begin{aligned}
& H=H^{(0)}+H^{(1)} \\
& M^{k 0}=\sum_{a=1}^{N}\left(q_{a}^{k} m_{a}-t k_{a}^{k}\right)+\frac{1}{2 c^{2}} \sum_{a, b=1}^{N} e_{a} e_{b} q_{b}^{k} u\left(q_{a b}\right),
\end{aligned}
$$

where

$$
\begin{aligned}
& H^{(0)}=\sum_{a=1}^{N}\left(m_{a} c^{2}+\frac{\mathbf{k}_{a}^{2}}{2 m_{a}}\right)+U^{(0)}, \quad U^{(0)}=\sum_{a<b}^{N} e_{a} e_{b} u\left(q_{a b}\right), \\
& H^{(1)}=-\sum_{a=1}^{N} \frac{\mathbf{k}_{a}^{4}}{8 m_{a}^{3} c^{2}}-\sum_{a<b}^{N} e_{a} e_{b}\left\{\frac{1}{2 c^{2} m_{a} m_{b}}\left[\mathbf{k}_{a} \mathbf{k}_{b} u\left(q_{a b}\right)+\left(\mathbf{k}_{a} \mathbf{q}_{a b}\right)\left(\mathbf{k}_{b} \mathbf{q}_{a b}\right) \frac{\mathrm{d} u\left(q_{a b}\right)}{q_{a b} \mathrm{~d} q_{a b}}\right]\right. \\
& \left.-\frac{A}{2 c^{2}}\left(\frac{\mathbf{k}_{a}}{m_{a}}-\frac{\mathbf{k}_{b}}{m_{b}}\right)^{2} u\left(q_{a b}\right)\right\},
\end{aligned}
$$

and $A=f^{\prime}(0)-1$. Specifically, $A=-1$ for the scalar and $A=0$ for the vector interactions. The latter in the massless case corresponds to the Darwin's Lagrangian for electromagnetic interaction. Expression (3.17) agrees with the post-Newtonian Hamiltonians obtained within various approaches [1]. 


\section{Statistical description}

Having obtained the Hamiltonian description of interacting particle system in the term of canonical particle variables we can define the relativistic partition function in the usual way

$$
Z=\frac{1}{N !} \int \mathrm{e}^{-\beta H} \prod_{a=1}^{N} \frac{\mathrm{d}^{3} k_{a} \mathrm{~d}^{3} q_{a}}{(2 \pi)^{3}}
$$

as an integral over the phase space of the particle system. But, according to (3.9), we need to define correctly the boundary conditions for the canonical coordinates $q_{a}^{i}$, while the physical variables $x_{a}^{i}$ vary into the volume $V$. It needs calculating the Jacobian $J=\partial\left(q_{a}^{i}, k_{a}^{i}\right) / \partial\left(x_{a}^{i}, k_{a}^{i}\right)$ by means of the expression (3.9). Next we can use the various approximations as in the nonrelativistic case.

Here we present only the result of the post-Newtonian approximation for the partition function corresponding to the Hamiltonian (3.14), (3.17). In this approximation Jacobian $J=1$. Using the general results of paper [10], we obtain in our case

$$
\begin{aligned}
& Z=Z^{\mathrm{id}} Q\left[1+R /\left(\beta m c^{2}\right)\right] \\
& R=\frac{15}{8} N-3 \beta \frac{A}{Q} \frac{\partial Q}{\partial \beta}, \quad Q=V^{-N} \int \mathrm{e}^{-\beta U^{(0)}} \prod_{a} \mathrm{~d}^{3} x_{a}
\end{aligned}
$$

where $m_{a}=m$ and $Z^{\text {id }}$ is the partition function of ideal gas.

Another way of calculating the partition function of the system can be based on the "field plus particle" Hamiltonians discussed in section 2. Paper [4] discusses the use of Gibbs approach, which is based on the Hamiltonian formulation of dynamics of such a system. Treating the field and particle variables on an equal level, the Liouville equation for distribution function and the partition function have been obtained. If the dynamics contains constraints, which have arisen in the system with vector-type interaction, we need to correctly reformulate the equations of statistical mechanics. Paper [4] demonstrates how one writes the Liouville equation for distribution function and the partition function for the system with constraints. Taking into account the nonlinear dependence of the instant form Hamiltonians (2.12), (2.17) and (2.20) on the physical fields, we cannot perform integration over fields without using the approximation scheme in the classical partition function. But it is demonstrated in [5] that the use of the front form of dynamics (given by $x^{0}=t+x^{3}$ ) allows us to exactly exclude the electromagnetic field variables from the partition function.

\section{References}

1. Gaida R. P. Quasirelativistic systems of interacting particles // Sov. J. Part. Nucl., 1982, vol. 13, p. 179.

2. Gaida R. P., Kluchkovsky Yu. B., Tretyak V. I. Forms of relativistic dynamics in the classical Lagrangian description of a system of particles // Theor. Math. Phys., 1983, 
vol. 55, No. 1, p. 372-384; Gaida R. P., Kluchkovsky Yu. B., Tretyak V. I. Threedimensional Lagrangian approach to the classical relativistic dynamics of directly interacting particles. - In: Constraint's Theory and Relativistic Dynamics. G. Longhi and L. Lusanna, eds. Singapore, World Scientific Publ., 1987, p. 210-241; Duviryak A., Shpytko V., Tretyak V. Isotropic forms of dynamics in the relativistic direct interaction theory // Cond. Matter Phys., 1998, vol. 1, No. 3(15), p. 463-512.

3. Crater H., Lusanna L. The rest-frame Darwin potential from the Lienard-Wiechert solution in the radiation gauge. SISSA e-preprint: hep-th/0001046, 2000; Alba D., Lusanna L. // Int. J. Mod. Phys. A, 1998, vol. 13, p. 2791.

4. Duviryak A., Nazarenko A. The Liouville equation for systems with constraints // J. Phys. Stud., 2000, vol. 3, No. 4, p. 399-408 (in Ukrainian).

5. Nazarenko A., Tretyak V. Classical relativistic systems of charged particles in the front form of dynamics and the Liouville equation // Cond. Matter Phys., 2000, vol. 3, No. 1(21), p. 5-22.

6. Rohrlich F. Classical Charged Particles: Foundations of Their Theory. AddisonWesley, New York, 1990; Parrott S. Relativistic Electrodynamics and Differential Geometry. Springer, New York, 1987.

7. Lusanna L. The $N$ - and 1-time classical descriptions of $N$-body relativistic kinematics and the electromagnetic interaction // Int. J. Mod. Phys., 1997, vol. 12, No. 4, p. 645722 .

8. Nazarenko A. Canonical realization of the Poincaré algebra for a relativistic system of charged particles plus electromagnetic field // Proceedings of Institute of Mathematics of NAS of Ukraine, 2000, vol. 30, Part 2, p. 343-349.

9. Currie D. G., Jordan J. F., Sudarshan E. C. G. Relativistic invariance and Hamiltonian theories of interacting particles // Rev. Mod. Phys., 1963, vol. 35, p. 350-375.

10. Tretyak V. On relativistic models in the equilibrium statistical mechanics // Cond. Matter Phys., 1998, vol. 1, p. 553-568. 
Класична релятивістична система $N$ зарядів. Гамільтонів опис, форми динаміки та статистична сума

\author{
А.Дувіряк, А.Назаренко, В.Третяк \\ Інститут фізики конденсованих систем НАН України, \\ 79011 Львів, вул. Свєнціцького, 1 \\ Отримано 3 жовтня 2000 p.
}

Розроблено процедуру редукції канонічних польових ступенів вільности для системи заряджених частинок з електромагнетним полем у гамільтоновому формалізмі з в'язями у першому порядку за константою взаємодії. Знайдено канонічну реалізацію алгебри Пуанкаре у термінах змінних частинок. Записано співвідношення між коваріянтними та фізичними змінними частинок. Також розглянуто систему частинок, які взаємодіють через скалярне та векторне масивні поля. Досліджено перше наближення за $c^{-2}$. Обговорюється застосування до обчислення статистичної суми системи взаємодіючих частинок.

Ключові слова: класична релятивістична механіка, форми релятивістичної динаміки, релятивістична статистична механіка, заряджені частинки

PACS: 03.30. $+p, 05.20 .-y$ 